\title{
Atypical Presentation of Ebstein's Anomaly in an adult patient - Magnetic resonance imaging assessment
}

\author{
Jose Luis Siller-Nava ${ }^{1}$, Jose Antonio Luna-Alvarez-Amezquita ${ }^{1}$, Joaquin Berarducci ${ }^{1}$, Javier Ivan Armenta-Moreno ${ }^{1}$, Jorge Luis Bermudez- \\ Gonzalez ${ }^{1}$, Roberto Cano-Zarate ${ }^{2}$ and Nilda Espinola-Zavaleta ${ }^{*}$
}

${ }^{1}$ Department of Nuclear Cardiology, National Institute of Cardiology Ignacio Chávez, Mexico City Mexico

${ }^{2}$ Department of Magnetic Resonance, National Institute of Cardiology Ignacio Chávez, Mexico City Mexico

\begin{abstract}
Ebstein's Anomaly (EA) is a complex and rare congenital cardiac disorder, representing 1\% of all congenital heart diseases; characterized by right ventricular myocardial abnormalities with a malformation of the tricuspid valve causing dilation and "atrialization". EA can have a wide variety of clinical presentations depending on the degree of the anatomical abnormality.

We present a 22-year-old male patient with EA diagnosed in 2008 at the age of 10, had no symptomatology until seven years later when abruptly he presented with dyspnea, syncope, chest pain, peripheral cyanosis and an abnormal heart rhythm. An electrocardiogram (ECG) with Wolff-Parkinson-White syndrome, and orthodromic tachycardia documented by a right posterolateral accessory pathway. TTE showed right ventricular atrialization of $67 \%$ and normal left ventricular systolic function (LVEF: 59\%), 12mm atrial septal defect with right to left shunt and severe tricuspid regurgitation with adequate right ventricular function. Surgical correction of the atrial septal defect and tricuspid valvuloplasty was performed.

Our patient had a milder presentation with no right ventricular dysfunction despite the severe atrialization, atrial septal defect, and severe tricuspid regurgitation. The particularity of this case is that patients with the previously described characteristics typically present with heart failure symptoms at much earlier stages in their life. This is a rare case, where tricuspid valvuloplasty was performed until adulthood.
\end{abstract}

\section{Introduction}

Ebstein's Anomaly (EA) is a complex and rare, representing only $1 \%$ of all congenital heart diseases [1]. It is characterized primarily by right ventricular myocardial development abnormalities, with a malformation of the tricuspid valve and myopathy of the right ventricle with dilation and "atrialization" [2], although it can also present with left heart abnormalities and associated atrial or ventricular septal defects [3].

EA can have a wide variety of clinical presentations depending on the degree of the anatomical malformation, ranging from severe symptomatic forms in neonates and young children to asymptomatic forms usually diagnosed incidentally in older patients. Presentation in neonatal and infant life often occurs with severe tricuspid regurgitation and anatomic right ventricular outflow tract obstruction [1]. In adults, arrhythmias are a common feature, presenting atrial fibrillation, flutter, or ectopic atrial tachycardia in up to $65 \%$ of patients and accessory pathways such as Wolff-Parkinson-White Syndrome (WPWS) in up to $45 \%$ of patients [2].

A diagnosis of EA should be suspected in cyanotic infants, children, and adults; in individuals with tricuspid regurgitation with or without heart failure and in those patients presenting with arrhythmias. Transthoracic echocardiography (TTE) is the recommended imaging modality for diagnosis, providing a platform for tricuspid valve (TV) leaflet characterization, determination of right heart size and function and evaluation of associated defects [3]. The diagnosis is confirmed by the identification of an apical displacement of the septal tricuspid valve leaflet of $\geq 8 \mathrm{~mm} / \mathrm{m} 2$ compared with the site of attachment of the anterior mitral valve leaflet, differentiating it from other congenital regurgitant lesions $[3,4]$.

Medical management and observation are often recommended for asymptomatic patients, and it can be successful for long periods of time with relatively high overall survival rates [2]. However, in patients who present increasing cardiomegaly or cyanosis, exercise intolerance and fatigue, paradoxical embolisms, or new onset atrial and ventricular arrhythmias, surgical intervention should be indicated for correction of the anatomical abnormalities presented by the patients [4].

\section{Case Report}

We present the case of a 22-year-old male patient with Ebstein's anomaly, who was diagnosed in 2008 at the age of 10 . Perinatal history showed that he was the product of a third pregnancy of normal evolution with vaginal delivery and no neonatal complications. Family history showed no relevant information. The patient was diagnosed with a heart murmur at the age of three, which was not clinically significant until seven years later when he presented with dyspnea and acrocyanosis in a routine check-up.

${ }^{\star}$ Correspondence to: Nilda Espinola-Zavaleta $\mathrm{MD}, \mathrm{PhD}$, Department of Nuclear Cardiology, National Institute of Cardiology, Ignacio Chavez, Mexico City Mexico, E-mail: nieza2001@hotmail.com

Key words: ebstein's Anomaly, congenital heart disease, echocardiography

Received: June 16, 2021; Accepted: June 22, 2021; Published: June 25, 2021 
In a further diagnostic approach, a TTE showed an Ebstein's anomaly with a right ventricular atrialization of $67 \%$ and left ventricular ejection fraction (LVEF) of 59\%, an atrial septal defect of $12 \mathrm{~mm}$ with right to left shunt and severe tricuspid regurgitation with mild right ventricular dysfunction (RVEF: $39 \%$ ). An electrocardiogram (ECG) with Wolff-Parkinson-White syndrome, and an orthodromic tachycardia documented by a right posterolateral accessory pathway that was managed medically with propaphenone $75 \mathrm{mg}$ twice a day.

Seven months after diagnosis he presented with syncope and chest pain, peripheral cyanosis, an abnormal heart rhythm, acropachy and a systolic murmur on tricuspid focus. A Magnetic Resonance Imaging (MRI) was made, which corroborated the echocardiographic data.

Ablation of right lateral and posterolateral accessory pathways was successful, with a remaining incomplete right bundle branch block (RBBB), and at four years of follow-up he remains asymptomatic without antiarrhythmic drugs.

Six months after discharge, the patient developed fatigue, orthopnea, lipothymia on two occasions and chest pain associated with moderate physical activity. After discussion with the surgical team, he was programmed for surgical correction. Pre-surgical echocardiogram reported right ventricular failure (RVEF: 25\%), type I diastolic dysfunction, severe tricuspid regurgitation with a dilated pulmonary artery trunk and preserved left ventricular systolic function. Pre-surgical MRI (Figure 1) confirmed an EA with $62 \%$ atrialization, Carpentier C and a Celermajer index of 0.53 and showed fibrosis in the atrialized portion of the right ventricle (Figure 1). The surgical procedure carried out was a closure of the atrial septal defect and tricuspid valvuloplasty with plication of the right ventricular atrialized portion and reduction of the anatomic annulus. In the $7^{\text {th }}$ day of post-surgical follow-up, the patient remained asymptomatic, with alternating node and sinus rhythms on the latest electrocardiographic evaluation. The patient is in under close observation in the out-patient clinic.

\section{Discussion}

Ebstein's anomaly (EA) is a rare congenital heart disease where patients rarely survive beyond adolescence without surgery or other medical procedures when patients present with symptoms at diagnosis. The mean age for diagnosis is 23.9 years with a standard deviation of
10.4. According to a cohort of 72 adult patients with EA the mean percentage of atrialization was $53 \%[5,6]$.

Our patient had a milder clinical presentation than would be expected for a severe atrialization of the right ventricle (62\%). He initially had no right ventricular dysfunction despite the percentage of atrialization, the atrial septal defect, and the severe tricuspid regurgitation. Patients with the previously described characteristics typically present with a NYHA functional class III/IV, even after surgical repairs, unlike our patient that had a NYHA functional class II [6].

Evaluation of EA in adults includes chest radiograph, electrocardiography, echocardiography, and magnetic resonance imaging. In chest radiographs, an abnormal silhouette is often found due to right heart enlargement and cardiomegaly with a "globular" appearance. Electrocardiogram shows right atrial enlargement, right bundle branch block, PR prolongation, low-voltage QRS in right-sided leads, and supraventricular tachycardia or atrial arrhythmias. WPWS is frequent in EA, and up to $30 \%$ of patients have multiple accessory pathways, as in this patient. Atrial arrhythmias are more frequent in adult patients than in neonates, these include atrial fibrillation, atrial flutter, and atrial tachycardia. Transthoracic echocardiography is the first choice for the evaluation of EA, it can confirm the diagnosis, estimate the severity, and recognize associated anomalies. MRI constitutes the "gold standard" for the assessment of right ventricular ejection fraction and the degree of fibrosis [7].

Commonly, the Celermajer index is used for echocardiographic evaluation in neonates and adults. It can be estimated with the information obtained from echocardiography and MRI [8]. Our patient with a Celermajer index of 0.53 lies within a milder form of the disease, despite having severe atrialization of the right ventricle, but without severe clinical manifestations for an unusually long period after the diagnosis [8].

Surgical correction of the anatomical abnormalities is usually recommended for patients between ages 2 to 5 years if the chance of good repair is likely. However, there have been studies that show relatively favorable prognosis in 4- year follow-up for older patients who had undergone surgical intervention, such as ours. Most of them had mild residual tricuspid regurgitation, but only $5 \%$ required rerepair intervention, with an overall early mortality of $2 \%$. Additionally,

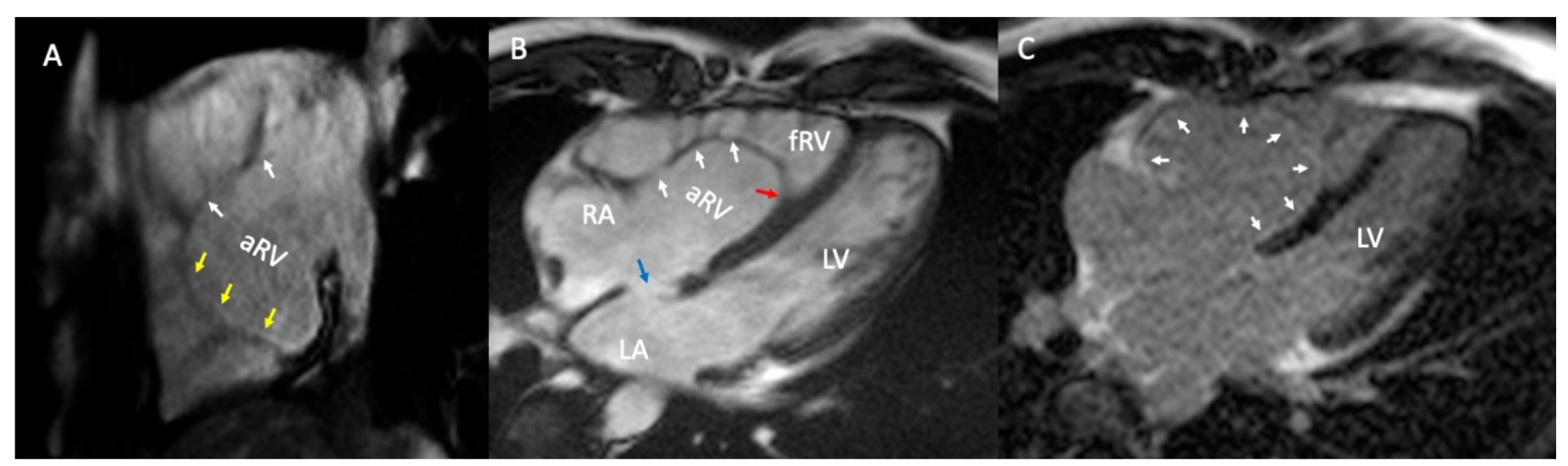

Figure 1. Magnetic Resonance Imaging. (A) Two chamber view, cine echo gradient, attachment of the posterior leaflet of the tricuspid valve (yellow arrows), anterior leaflet appears elongated (white arrows). (B) Four chamber view, cine echo gradient, attachment of the septal leaflet of the tricuspid valve involving $65 \%$ of the right ventricle (red arrow), with redundant anterior leaflet (white arrows) and an ostium secundum atrial septal defect (blue arrow). (C) Four chamber view, late gadolinium enhancement in the atrialized portion of the right ventricle (white head arrows)

Abbreviations: LV-left ventricle; RA-right atrium; LA-left atrium; aRV-atrialized right ventricle; fRV-functional right ventricle. 
the presence of a sinus rhythm or an accessory pathway, as shown in this patient, is associated with lower mortality rates [4].

Future efforts in the investigation of current severity scores and indexes must be made to better classify patients with EA and improve clinical outcomes and clarify the prognosis for patients.

\section{Conclusion}

The particularity of this case is based upon the fact that patients with severe atrialization of the right ventricle, usually present with heart failure symptoms at much earlier stages in their life. His initial diagnosis was made due to the presence of a persistent heart murmur and WPWS, which states the importance for the clinician to have high suspicion rates of EA in patients with this arrhythmia. Surgical interventions are required for improving clinical outcomes and survival rates at adolescence and even sooner. This is a rare case where tricuspid valvuloplasty was performed until adulthood with a favorable outcome. The importance of documenting cases like ours is to highlight the clinical course of adults with congenital heart diseases that is not represented in the literature.

\section{Funding}

None.

\section{Disclosures}

None.

\section{Conflict of Interest}

None.

\section{Acknowledgments}

None.

\section{References}

1. Sherwin E, Abrams D (2017) Ebstein Anomaly. Card Electrophysiol Clin 9: 245-254 [Crossref]

2. Holst K, Connolly H, Dearani J (2019) Ebstein's Anomaly. Methodist Debakey Cardiovasc J 15: 138-144. [Crossref]

3. Qureshi M, O'Leary P, Connolly H (2018) Cardiac imaging in Ebstein anomaly. Trends Cardiovasc Med 28: 403-409. [Crossref]

4. Sainathan S, Silva L, Silva J (2020) Ebstein's anomaly: contemporary management strategies. J Thorac Dis 12: 1161-1173. [Crossref]

5. Tourmousoglou C, Bogossian H, Ninios V, Ninios E (2019) A rare case of Ebstein's anomaly with left ventricular noncompaction. Asian Cardiovasc Thorac Ann 27: 208209. [Crossref]

6. Attie F, Rosas M, Rijlaarsdam M, Buendia A, Zabal C, et al. (2000) The adult patient with Ebstein anomaly. Outcome in 72 unoperated patients. Medicine (Baltimore) 79 : 27-36. [Crossref]

7. Dearani JA, Mora BN, Nelson TJ, Haile DT, O'Leary PW (2015) Ebstein anomaly review: what's now, what's next? Expert Rev Cardiovasc Ther 13: 1101-9. [Crossref]

8. Aleksandra C, Olga T, Agnieszka BR, Anna K, Justyna RT, et al. (2019) Severity Scores for Ebstein Anomaly: Credibility and Usefulness of Echocardiographic versus Magnetic Resonance Assessments of the Celermajer Index. Can J Cardiol 35: 1834 1841. [Crossref]

Copyright: (C2021 Siller-Nava JL. This is an open-access article distributed under the terms of the Creative Commons Attribution License, which permits unrestricted use, distribution, and reproduction in any medium, provided the original author and source are credited. 